\title{
Conversion Total Knee Arthroplasty after Failed High Tibial Osteotomy
}

\author{
Sang Jun Song, MD, Dae Kyung Bae, MD, Kang Il Kim, MD, and Chung Hwan Lee, MD \\ Department of Orthopedic Surgery, Kyung Hee University College of Medicine, Seoul, Korea
}

\begin{abstract}
Clinical results of high tibial osteotomy (HTO) deteriorate over time despite the initial satisfactory results. Several knees may require a conversion to total knee arthroplasty (TKA) because of failure such as the progression of degenerative osteoarthritis and the loss of the correction angle. It is important to know the long-term survival rate and common reason of failure in HTO to inform patients of postoperative expectations before surgery and to prevent surgical errors during surgery. In addition, it has been reported that clinical and radiological results, revision rate, and complication rate were poorer than those in patients without a previous HTO. There are few review articles that describe why conversion TKA after HTO is surgically difficult and the results are poor. Surgeons have to avoid the various complications and surgical errors in this specific situation. We would like to present the considering factors and technical difficulties during conversion TKA after HTO with a review of the literature. We could conclude through the review that the correction of deformity, lower amount of tibial bone resection, and sufficient polyethylene insert thickness, restoration of the joint line height, and adequate ligament balancing can be helpful in overcoming the technical challenges encountered during TKA following HTO.
\end{abstract}

Keywords: Knee, Osteoarthritis, Osteotomy, Arthroplasty

\section{Introduction}

High tibial osteotomy (HTO) is a common surgical option for osteoarthritic (OA) knees with varus deformity, especially in young and active patients ${ }^{1,2)}$. However, clinical results of HTO deteriorate over time despite the initial satisfactory results. Previous studies have reported the 5-year survival rate of HTO ranging from $71 \%$ to $95 \%$ and the 10 -year survival rate ranging from $51 \%$ to $98 \%^{3-10)}$. Several knees may require a conversion to total knee arthroplasty (TKA) because of failure such as the progression of degenerative osteoarthritis and the loss of the correction angle. Various factors have been reported to be associated with

Received October 12, 2015; Revised November 4, 2015;

Accepted November 6, 2015

Correspondence to: Dae Kyung Bae, MD

Department of Orthopedic Surgery, Kyung Hee University College of Medicine, 26 Kyungheedae-ro, Dongdaemun-gu, Seoul 02447, Korea Tel: +82-2-958-8348, Fax: +82-2-964-3865

E-mail: bdkyung@khmc.or.kr

This is an Open Access article distributed under the terms of the Creative Commons Attribution Non-Commercial License (http://creativecommons.org/licenses/by-nc/4.0/) which permits unrestricted non-commercial use, distribution, and reproduction in any medium, provided the original work is properly cited. the survival of an HTO, including age, gender, body mass index, preoperative range of motion (ROM), OA grade, and postoperative correction angle ${ }^{6,7,10)}$. It is necessary to know the long-term survival rate, affecting factors and common reasons of failure in HTO to prevent those kinds of errors and elucidate proper indications. However, controversies regarding the factors affecting the survival rate of HTO still exist ${ }^{6,7,9,11)}$.

It has been reported that clinical and radiological results, revision rate, and complication rate in conversion TKA after HTO are poorer than those in patients without a previous HTO. It may be technically more demanding than a primary TKA due to the difficulty of surgical approach, ligamentous imbalance, and the anatomical distortion of the proximal tibial metaphysis after $\mathrm{HTO}^{12)}$. However, it is still a debating issue whether HTO can have any harmful effects on the result of a subsequent TKA. For a successful conversion TKA, accurate positioning of implants and proper balancing are required. Surgeons need to avoid various complications and surgical errors in this specific situation. We would like to discuss the considering factors for technical difficulties during the conversion TKA after HTO with a review of the literature. 


\section{Long-Term Survival Rate of HTO and Factors Affecting the Survival Rate}

Previous studies have reported a wide range of mid- and longterm survival rates ${ }^{3-10}$. Differences in results among previous studies may have been caused by wide heterogeneity in terms of indication, severity of varus deformity prior to HTO, surgical techniques, fixatives, and rehabilitation protocols ${ }^{6}$. Comparison and pooling of data from previous studies on HTO are also challenging due to different evaluation systems and small cohort sizes ${ }^{4}$. The survivorship analysis and comparison of survival rates are useful statistical methods for comparing multiple cohort groups with different follow-up periods. Although most studies have shown that good short- and mid-term survival rates can be achieved, these rates deteriorated over time (Table 1$)^{13-17)}$. The annual failure rates markedly increased about 10 years postoperatively. In our recent long-term survivorship analysis after closed wedge HTO, the survival curve showed a good mid-term survival rate, but the annual failure rate markedly increased after 11 years postoperatively (Bae DK, Unpublished Data) (Fig. 1). The open wedge HTO using a locking plate is currently popular due to its initial stability and surgical simplicity. TomoFix (Depuy, Warsaw, IN, USA) was introduced a decade ago ${ }^{18)}$ and sufficient survivorship analyses could be conducted; therefore, we expect publications on the long-term survivorship analysis of open wedge HTO using a modern technique and a locking plate. The possibility of conversion TKA should be considered after HTO because degenerative OA can progress postoperatively, and it should be taken into account to determine the timing of HTO in young patients.

\section{Reason of Failure after HTO}

Intraoperative complications of HTO include neurovascular injury, under- or overcorrection of the lower limb alignment, fracture of the opposite cortical hinge, intraarticular fracture, and unintended change of the tibial posterior slope angle. Postoperative complications include nonunion or delayed union of the osteotomy site, change of the patellar height, and infection. Most complications are caused by surgical errors and can be avoided with a meticulous surgical technique. In our review of more than

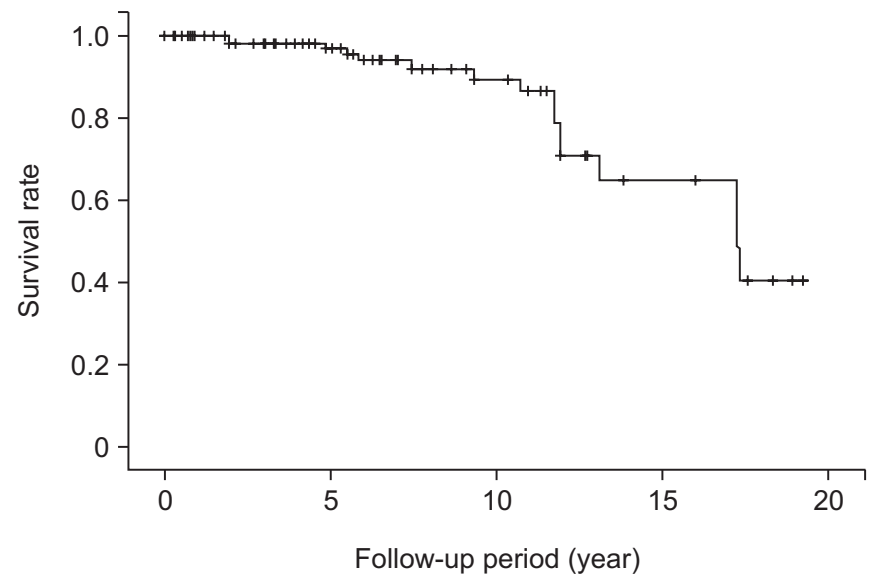

Our recent research, KOA, 2014

Fig. 1. Long-term survivorship analysis following closed wedge high tibial osteotomy using miniplate staple in our hospital. Although the survival curve shows good mid-term survival rate, the annual failure rate was markedly increased after 11 years postoperatively. KOA: Annual meeting of Korean Orthopaedic Association.

Table 1. Survival Rate in Previous Studies

\begin{tabular}{|c|c|c|c|c|c|}
\hline HTO & Author & Year & Fixatives & 5 -year & 10-year \\
\hline \multirow[t]{7}{*}{ Closed } & Akizuki et al. ${ }^{3)}$ & 2008 & Giebel plate & & 97.6 \\
\hline & Efe et al. ${ }^{6}$ & 2011 & AO plate & 93 & 84 \\
\hline & Hui et al. ${ }^{7)}$ & 2011 & Krakow staple & 95 & 79 \\
\hline & van Raaij et al. ${ }^{10)}$ & 2008 & Step staple & 90 & 75 \\
\hline & Sprenger and Doerzbacher ${ }^{13)}$ & 2003 & AO plate & 86 & 74 \\
\hline & Flecher et al. ${ }^{14)}$ & 2006 & Staple or AO plate & 94.8 & 92.8 \\
\hline & Gstottner et al. $^{15)}$ & 2008 & Staple & 94 & 79.9 \\
\hline \multirow[t]{4}{*}{ Open } & Bonasia et al. ${ }^{4)}$ & 2014 & Puddu plate & 98.7 & 75.9 (7 yr) \\
\hline & DeMeo et al. ${ }^{5)}$ & 2010 & Puddu plate & & $70(8 \mathrm{yr})$ \\
\hline & Schroter et al. ${ }^{16)}$ & 2011 & Aescula plate & $77(1 \mathrm{yr})$ & \\
\hline & Bode et al. ${ }^{17)}$ & 2013 & TomoFix & 96 & \\
\hline
\end{tabular}

HTO: high tibial osteotomy. 
300 HTOs of recent 10 years (Bae DK and Song SJ, Unpublished Data), there was no case of neurovascular injury, nonunion, and infection. However, the avoidance of under- or overcorrection, cortical or plateau fracture, change in the tibial posterior slope angle in every knee may not be easy.

Various methods can be used to determine the accurate correction angle and wedge size, including radiographic measurements and planning, intraoperative measurements with the help of a cable method, grids with lead-impregnated reference lines, or a jig system. The application of navigation system on HTO can be useful because it can be an intraoperative protractor for alignment with weight bearing or dynamic simulation ${ }^{19,20)}$. It can also calculate the length of the osteotomy, and the position of cortical hinge can be adjusted to avoid cortical disruption in computerassisted HTO. Fracture of the opposite cortical hinge and intraarticular fracture can be encountered during HTO especially when the large correction angle and long wedge height are required. It can disrupt stability, healing at the osteotomy site, and congruence of the articular surface ${ }^{21}$. In these knees, the use of locking compression plates or extra screws should be considered to provide stable fixation and reduce the risk of loss of correction angle and nonunion ${ }^{21)}$.

Lateral closed wedge osteotomy causes proximation of the tibial tuberosity to the joint line with wedge resection during the procedure, which theoretically causes patella alta. However, patella baja is clinically more common. This is probably due to postoperative immobilization and scarring, or patellar tendon slackness resulting from the reduction of metaphyseal thickness ${ }^{22}$. Open wedge osteotomy relatively shortens the patellar tendon above the joint line, which results in patella baja. With the recent advancement in fixatives and internal fixation techniques, the incidence of peripatellar adhesion and patella baja has been decreased although long-term cast immobilization frequently increased the risk in the past.

The HTO can result in changes in the tibial posterior slope angle. In general, it is well known that medial open wedge HTO increases posterior slope whereas lateral closed wedge HTO decreases it. Most surgeons try to restore the original slope with various methods. Positioning the extractor or fixation plate on the posterior side, sufficient posterior soft tissue releasing, and asymmetrical opening of the posterior wedge were introduced to avoid the increase in posterior slope in open wedge osteotomy ${ }^{23)}$. It is important to perform the proximal and distal osteotomy parallel to the articular surface and to position the hinge axis perpendicular to the osteotomy plane in order to maintain the posterior slope in closed wedge $\mathrm{HTO}^{19,20)}$.

\section{Previous Results of Conversion TKA}

It has been reported that the clinical and radiological results, revision rate, and complication rate were poorer than those in patients without a previous $\mathrm{HTO}^{24-32}$ or similar (Table 2) ${ }^{33-40)}$. The variety of previous results are reasoned by the differences in the anatomical deformity and ligament status prior to conversion TKA, type of prosthesis, surgical techniques, control group, and the duration of follow-up period. van Raaij et al. ${ }^{41)}$ reported that the previous HTO does not compromise subsequent TKA with a systematic review and the low quality of evidence precludes solid clinical conclusions. Previous studies reporting the less satisfactory results warn the high risk of complications and revision due to aseptic loosening, infection, stiffness, patellar instability, etc ${ }^{28,42}$. Cameron and Park $^{29)}$ reported poor results of $17.5 \%$ in the conversion TKA after failed HTO at 2 to 15 years follow-up and an overall complication rate of $11.2 \%$. Parvizi et al. ${ }^{28}$ reported poorer Knee Society pain score in the conversion TKA after HTO group with a higher incidence of aseptic loosening in a 15-year follow-

Table 2. Various Results of Conversion Total Knee Arthroplasty (TKA) Following Previous High Tibial Osteotomy Compared to Primary TKA according to Authors

\begin{tabular}{|c|c|c|c|c|c|c|c|}
\hline \multicolumn{4}{|c|}{ Less satisfactory } & \multicolumn{4}{|c|}{ No difference } \\
\hline No. & Author & Journal & Year & No. & Author & Journal & Year \\
\hline 1 & Haslam et al. $^{24)}$ & J Arthroplasty & 2007 & 1 & Meding et al. ${ }^{33)}$ & Clin Orthop Relat Res & 2011 \\
\hline 2 & Madan et al. ${ }^{25)}$ & Bull Hosp Jt Dis & $2002-2003$ & 2 & Kazakos et al. ${ }^{34)}$ & Arch Orthop Trauma Surg & 2008 \\
\hline 3 & Niinimaki et al. ${ }^{27)}$ & Arch Orthop Trauma Surg & 2014 & 3 & Haddad and Bentley ${ }^{35)}$ & J Arthroplasty & 2000 \\
\hline 4 & Parvizi et al. ${ }^{28)}$ & J Bone Joint Surg Am & 2004 & 4 & Bastos Filho et al. ${ }^{36)}$ & Int Orthop & 2013 \\
\hline 5 & Cameron and Park ${ }^{29)}$ & Orthopedics & 1996 & 5 & Toksvig-Larsen et al. ${ }^{37)}$ & J Bone Joint Surg Br & 1998 \\
\hline 6 & Windsor et al. ${ }^{30)}$ & J Bone Joint Surg Am & 1988 & 6 & Amendola and Bonasia ${ }^{38)}$ & Int Orthop & 2010 \\
\hline 7 & Noda et al. ${ }^{31)}$ & J Orthop Sci & 2000 & 7 & van Raaij et al. ${ }^{39)}$ & BMC Musculoskelet Discord & 2007 \\
\hline 8 & Mont et al. ${ }^{32)}$ & Clin Orthop Relat Res & 1994 & & & & \\
\hline
\end{tabular}


up study. Kazakos et al..$^{34)}$ reported comparable results at 4.5 years of follow-up in spite of a statistically significantly higher rate of intra- and postoperative complications, including impingement between the tibial stem and lateral tibial cortex, patellar baja, and patellar subluxation. Haddad and Bentley ${ }^{35}$ also reported comparable results at 6-year follow-up although patella baja was present in $60 \%$ of patients in the conversion TKA group. In spite of the heterogeneity of the previous results, most surgeons agree with the consensus that the skin and soft tissue status, combined deformity, and ligamentous distortion around the knee after previous HTO can make the surgical procedures more difficult ${ }^{12,222}$. There are lots of considering factors and technical difficulties to avoid the various complications and surgical errors in the conversion TKA after HTO.

\section{Considering Factors Regarding Surgical Difficulties and Risks in Conversion TKA}

The skin and soft tissue scarring, patella baja, and limited knee motion make the surgical approach difficult. The anatomical deformity and bony distortion of the proximal tibial metaphysis after HTO make the conversion TKA difficult ${ }^{12)}$. Preoperative deformities include varus/valgus and rotational deformity, a change in the tibial slope angle, and the medial displacement of the tibial anatomical axis ${ }^{12,42}$. Restoration of the normal joint line with careful determination of the tibial bone resection level and proper soft tissue balancing are also required ${ }^{12}$. We describe the considering factors including surgical approach, anatomical distortion and deformities, soft tissue imbalance, and issues of selection of prosthesis types in order.

\section{Surgical Approach}

Previous surgical scars for HTO can be transverse or longitudinal in the medial or lateral aspect of the proximal tibia. Transverse scars can be ignored safely, but multiple longitudinal scars should be handled cautiously. The knee joint is vulnerable to multiple parallel incisions. The lateral flap is far more vulnerable since the blood supply and lymph fluid drainage are mainly located on the medial side. If there is a previous operation scar, the skin incision can be made from the far lateral previous incision scar and approached through medial arthrotomy. According to the surgeon's preference, lateral arthrotomy can be performed in severe valgus deformity patients or when the operator is familiar to the lateral approach. The most common surgical challenge identified in conversion TKA is everting the patella. It can be caused by infrapatellar and periosteotomy site adhesion or patellar baja ${ }^{12}$. Therefore, infrapatellar release was performed carefully in a stepwise manner in order to prevent a patellar tendon injury. If the patellar eversion is still difficult, it can require a special approach technique, such as early release of the lateral retinaculum and patellofemoral ligament, Rectus snip, V-Y plasty of quadriceps, and tibial tubercle osteotomy (TTO $)^{43)}$. Bastos Filho et al ${ }^{36)}$ reported the incidence of additional procedures for surgical approach such as TTO or Rectus snip was $25 \%$ because of the difficult patellar eversion in conversion TKA after HTO. Quadriceps snip and V-Y plasty are commonly adopted and were necessary in $23 \%$ of patients reported by Gill et al. ${ }^{44)}$ and in $40 \%$ of patients reported by Toksvig-Larsen et al. ${ }^{37)}$. But, V-Y plasty weakens the extensor mechanism and leads to greater patella baja or extension lag. The TTO provides wide knee joint exposure and allows bone-to-bone healing and maintains the blood supply to the patella and surrounding soft tissue. However, postoperative extensor lag, proximal migration of the tibial tubercle, and tibial fracture can occur ${ }^{45-47}$. In general, lateral retinacular release is not required for proper exposure for primary TKA. The number of knees that require a lateral retinacular release is slightly greater in the group with a previous HTO than in the group without a previous HTO although the difference is not significant ${ }^{43}$. Because the previous closed wedge HTO can shorten the distance of the tibial tubercle from the joint line and can make exposure of the proximal part of the tibia and lateral dislocation of the patella more difficult, surgeons need to be cautious about the risk of avulsion of the tibial tubercle. Treuter et al. ${ }^{48}$ described the surgical advantages of HTO include it is performed below the tibial osteotomy, causes no problems concerning patella height, and necessitates no additional procedure for exposure of the knee joint ${ }^{48}$.

We usually use the midline skin incision and medial parapatellar approach with careful infra- and peripatellar release with no additional procedure. We think that it may be caused by the low incidence of patellar baja with early rehabilitation after closed wedge HTO. After HTO, dynamic changes to the patellofemoral joint, such as patella baja, alteration of the $\mathrm{Q}$ angle and patellar subluxation, are associated with the fixation methods of osteotomy and postsurgical rehabilitation method ${ }^{12,49}$. Inadequate fixation of the osteotomy site can delay rehabilitation and increase the likelihood of those dynamic changes. Patellar tendon may be shortened by excessive new bone formation during osteotomy healing. Windsor et al. ${ }^{30)}$ reported that the average Insall-Salvati ratio was 0.7 preoperatively, and 32 out of 45 cases were less than 0.8. In our previous research (Bae DK, Unpublished Data), the average Insall-Salvati ratio was 0.86 before TKA and 0.85 at the 
last follow-up. There were 5 knees with less than 0.8 before conversion TKA among 32 knees.

Fixatives for the osteotomy site can be remained if it does not irritate soft tissue and cause pain; however, if it interrupts the placement of the tibial components, it should be removed ${ }^{43)}$. The removal procedure can be performed simultaneously during the conversion TKA procedure, or with an interval of time prior to it. The staples or screws can be safely removed through the previous skin incision during simultaneous conversion TKA. For the removal of a bulky plate using a separate incision and soft tissue dissection besides the anterior midline incision, a staged procedure with an interval of 4-6 weeks would be safe, considering the time for soft tissue healing.

\section{Anatomical Deformities}

Because a coronal or rotational deformity of the tibial plateau and a change in the posterior slope angle can occur after closed and open wedge $\mathrm{HTO}^{43)}$, conversion TKA requires good understanding of these deformities. Most malunion can be corrected intra-articularly at the time of TKA because the metaphyseal deformities are close to the joint line. However, in severe deformities, this can lead to soft tissue imbalance and ligamentous distortion should be considered. The resected bone surfaces can be unusual in shape in severely deformed knees, thus the surgeons need to be familiar with them (Fig. 2). For rotational deformity, the correction of tibial axial malunion by modifying the position of the tibial component can be dangerous because it affects both tibiofemoral and patellofemoral kinematics. For severe malunion, derotational osteotomy or medial transfer of the tibial tuberosity can be proposed to improve the patellofemoral kinematics ${ }^{22)}$.
In addition, there can be translational and meta-diaphyseal mismatch of the tibia. In general, the tibial anatomical axis may pass the medial or the antero-medial point on the tibial plateau after HTO, and the tibial component stem can come in contact with the lateral tibial cortex ${ }^{50)}$. This problem can be solved by undersizing or medial placement of the tibial component and the use of an offset stem $^{50)}$.

A tibial metaphyseal bone loss after closed wedge HTO requires less amount of tibial bone resection at the time of conversion $\mathrm{TKA}^{12)}$. Meding et al. ${ }^{43)}$ reported that the average amount of tibial bone resection was $3.3 \mathrm{~mm}$ in a conversion TKA group with a previous HTO and $7.5 \mathrm{~mm}$ in a control group without a previous HTO. The proximal tibial bone should be resected conservatively first, keeping in mind the previous metaphyseal bone loss and restoration of the original joint line.

\section{Ligament Balance}

Medial and lateral imbalance is frequently observed after bone resection using a measured resection technique in cases where a previous HTO produced an extra-articular deformity and influenced soft tissue tension. A femoral and tibial bone resection perpendicular to the mechanical axis results in an asymmetric gap, which produces the so-called resection laxity and increases the preexisting imbalance ${ }^{22)}$. At the time of conversion TKA, undercorrection and varus recurrence or overcorrection and excessive valgus deformity can be encountered. In cases of varus recurrence with medial tibial plateau wear and moderate metaphyseal malunion, a medial release is performed as much as necessary. The cutoff value for correctable deformities is not always predictable during the preoperative evaluation, and preoperative stress
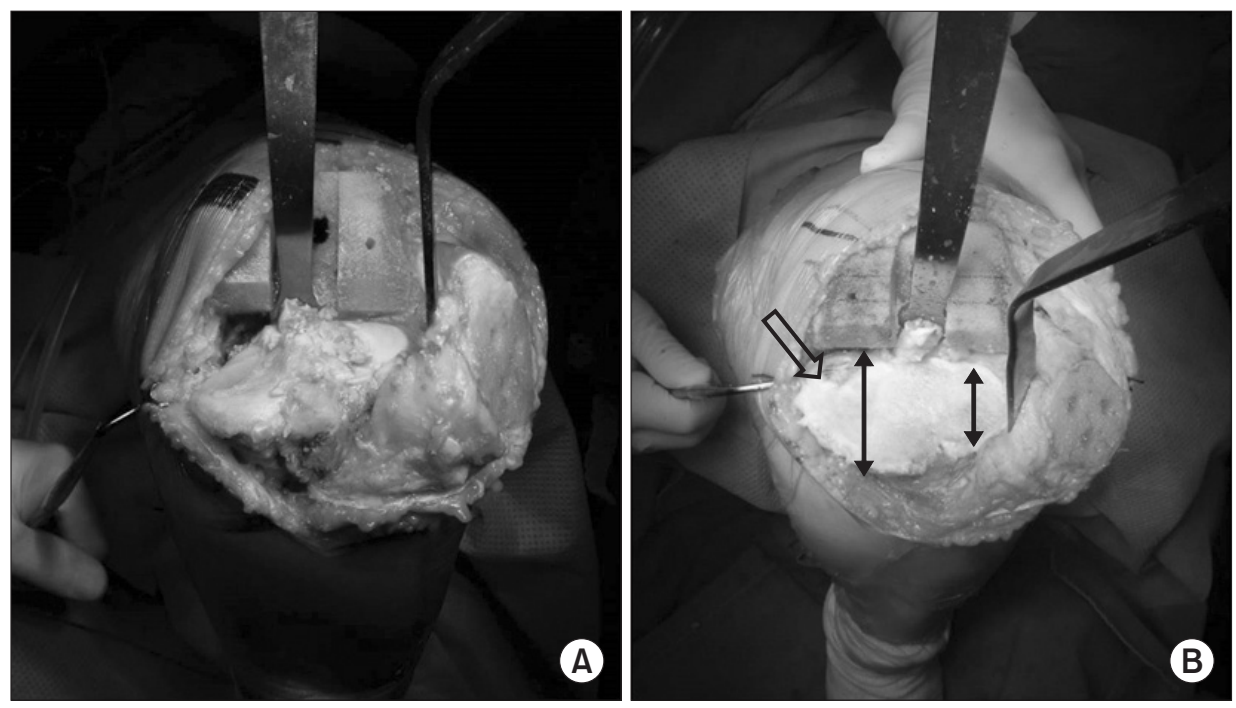

Fig. 2. Anatomical distortion of proximal tibia after closed wedge high tibial osteotomy (HTO) and prepared surface of the tibia. (A) The severe coronal deformity of the tibial plateau and a change in the posterior slope angle after HTO can lead to unusual shape of the resected bone surfaces of the tibia. (B) This patient has a large difference in the anteroposterior length (line segment) between the cut surfaces of the medial and lateral tibial condyles. The unresected posterior condyles are shown as well (vacant arrow). 
radiographs can be helpful ${ }^{22}$. In cases of overcorrected valgus deformity, it is more difficult to balance properly. The postoperative change of lower extremity alignment can cause disorder of the collateral ligament and posterior cruciate ligament (PCL). Bone defect of the lateral tibia plateau can exist, and the femoral articular rotational translation effect during knee flexion following the valgus deformity can exist as well. It may also occur due to lateral soft tissue retraction after closed wedge HTO or medial collateral ligament insufficiency after open wedge HTO (Fig. 3). Whatever the reasons may be, surgeons should not only get the proper mediolateral and flexion-extension gap balancing but also avoid over-release of the medial or lateral structure and instability. Aglietti et al. ${ }^{51)}$ performed minimal tibial resection and compensated soft tissue imbalance through PCL release or sacrificing and pie-crusting of the lateral capsule.

Bastos Filho et al. ${ }^{36)}$ reported different incidences of medial or lateral release in conversion TKA after open versus closed wedge HTO. Their incidences of medial and lateral release were 91.5\% and $46.0 \%$ in conversion TKA after open wedge HTO, and they were $55.5 \%$ and $57.2 \%$ in conversion TKA after closed wedge HTO. However, we think that ligament balancing during a conversion TKA is not as difficult as it is known when the conversion TKA is performed due to progression of degenerative osteoarthritis with minimally altered anatomy of the proximal tibia and without previous HTO complications.

\section{Issues of Selection of Prosthesis Types}

Many authors recommend the use of the PCL substituting prosthesis for conversion TKA after HTO because of PCL contracture, insufficiency, and postoperative stiffness ${ }^{52,53)}$. They claimed that it was difficult to maintain the appropriate tension of the PCL and balance the flexion and extension gap because of the tibial bone defect and the relaxation of the PCL. Hernigou et al. ${ }^{52)}$ reported good results after conversion TKA with a posteriorstabilized prosthesis. Kleinbart et al. ${ }^{54)}$ demonstrated that PCLs obtained from OA knees were characterized by distinct histological degenerative changes which were markedly different from normal age-related changes. Degenerative changes in the PCL might be accelerated in knees subjected to HTO, even though the tension and appearance of the PCL at the time of surgery seem to be intact. A high rate of femoral and tibial component loosening was reported after conversion TKA using a PCL retaining prosthesis ${ }^{43)}$. Akasaki et al. ${ }^{53)}$ reported that the Knee Society score, stability, ROM in PCL retaining TKA were inferior to PCL substituting TKA. They performed closed wedge HTO that could result in a decrease in posterior slope that caused hyperextension and overload on the PCL. Hernigou et al. ${ }^{52)}$ argued that patients even with an overcorrected HTO had improvements in function and pain using a posterior-stabilized prostheses and that the degree of deformity had no impact on the longevity of conversion TKA.

In addition, routine patella resurfacing has been recommended
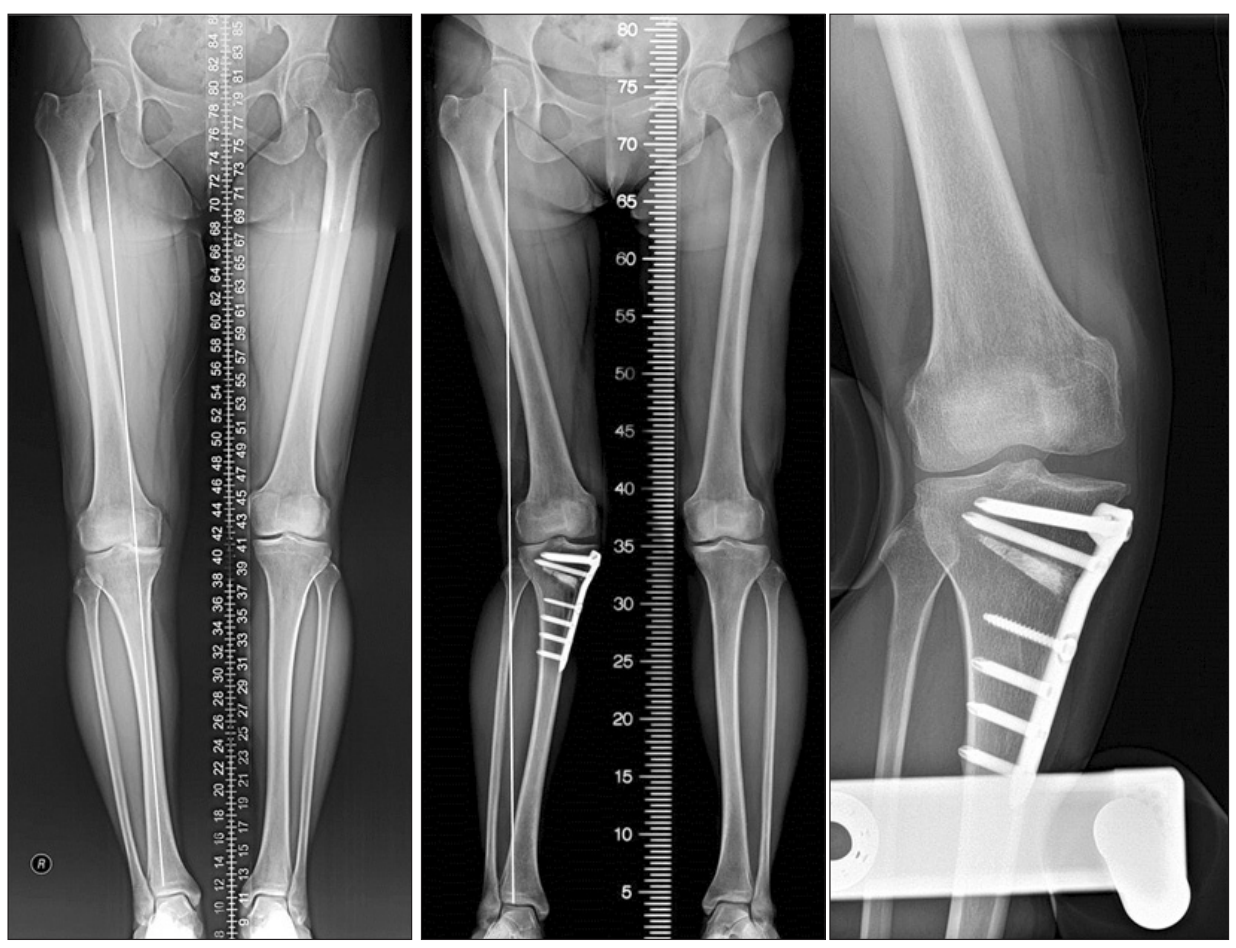

Fig. 3. Valgus overcorrection after open wedge high tibial osteotomy (HTO) with ligament insufficiency. It is suspected that the medial collateral ligament injury after open wedge HTO aggravates valgus deformity after surgery. 
because of the high risk of anterior knee pain and high revision rate for secondary resurfacing of the patella if it is not resurfaced in the conversion TKA after $\mathrm{HTO}^{38)}$. Amendola and Bonasia ${ }^{38)}$ reported the results of 29 consecutive TKAs after failed HTO. Three patients had persistent anterior pain, which required a further arthroplasty of the patella performed, respectively, at 18, 19, and 27 months after the index arthroplasty, with a marked clinical improvement. Considering the possible change of the patellar height and peripatellar soft tissue scarring after failed HTO, it is understandable that the patellar resurfacing procedure is safer than patellar nonresurfacing during conversion TKA.

If nonunion after HTO occurred, treatment of the nonunion is necessary while performing conversion TKA. Complete removal of the fibrous tissue, autogenous bone graft using the resected bone, and long-stem tibial prosthesis should be considered for bone union and stable component fixation.

Semi-constrained type prosthesis can be considered in excessive varus or valgus deformity, unable to obtain accurate ligament balancing for soft tissue imbalance after intra-articular correction of extra-articular deformity.

\section{Our Recent Research about Mid-Term Clinical and Radiographic Results in Conversion TKAs Follow- ing Closed Wedge HTO}

A total of 32 conversion TKAs (29 patients) with an average follow-up period of 6.2 years were retrospectively reviewed in our hospital (Bae DK, Unpublished Data). The clinical results were evaluated using the Knee Society knee and function score, the Western Ontario and McMaster Universities (WOMAC) score, Kujala scores, and ROM. The radiographic results were evaluated using the femorotibial angle, the method employed by the American Knee Society, Insall-Salvati ratio, the joint line height (JLH), and the amount of tibial bone resection. The mean knee score was improved from 49.1 to 90.6 , and the mean function score was improved from 51.2 to 88.8. The WOMAC averaged 57.5 preoperatively and 9.3 at the last follow-up. The mean Kujala score was improved from 52.7 to 83.8 . The mean preoperative and postoperative ROM were $118.9^{\circ}$ and $129.1^{\circ}$, respectively. The preoperative femorotibial angle averaged varus $4.1^{\circ}$ preoperatively and valgus $6.0^{\circ}$ postoperatively. The position of the components in every knee was within the permissible range. The mean Insall-Salvati ratio was 1.07 preoperatively and 1.13 postoperatively. The average JLH was $13.2 \mathrm{~mm}$ preoperatively and 14.9 $\mathrm{mm}$ at the final follow-up. The average amount of tibial bone resection was determined to be $3.2 \mathrm{~mm}$. The median thickness of polyethylene insert was $10 \mathrm{~mm}$ (Fig. 4). The previous closed wedge HTO itself had no detrimental effect on the mid-term outcome of the subsequent TKA. Meticulous surgical technique with careful consideration of deformity correction, balancing, and restoration of the JLH and patellar height can bring satisfactory results in conversion TKA after closed wedge HTO.
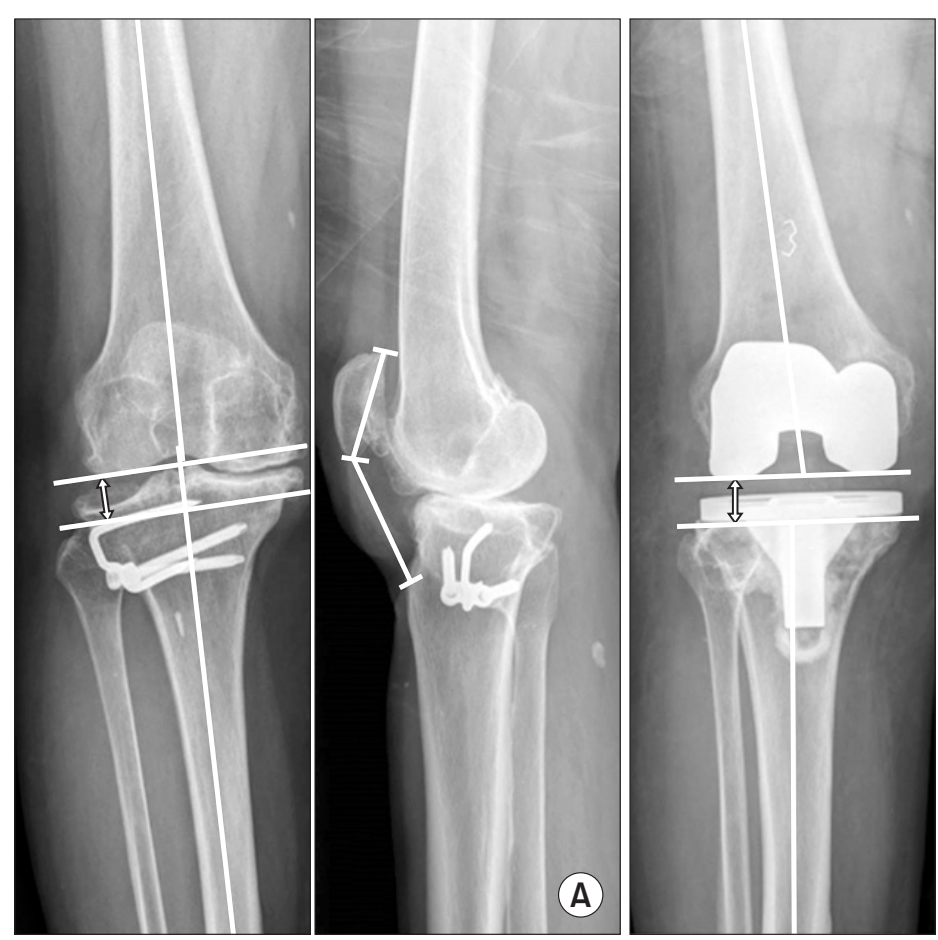

Fig. 4. Preoperative radiographs of the right knee of a 64-year-old woman with a prior history of high tibial osteotomy 14 years ago. The joint line height and Insall-Salvati ratio were well preserved after conversion total knee arthroplasty (15.3 mm vs. $16.6 \mathrm{~mm}$ and 1.21 vs. 1.19 , respectively). The radiographically measured amount of tibial bone resection was $3.1 \mathrm{~mm}$. 
In conclusion, several knees after HTO may require a conversion TKA because of failure such as the progression of degenerative osteoarthritis and the loss of the correction angle. It is generally accepted that TKA after closed wedge HTO is technically more difficult. Surgeons need to consider the various factors affecting the technical difficulty during conversion TKA, including surgical approach, anatomical deformities, ligament imbalance, and selection of prosthesis types. The correction of deformity, lower amount of tibial bone resection, sufficient polyethylene insert thickness, restoration of the joint line height, and adequate ligament balancing can be helpful in overcoming the technical challenges encountered in TKA following HTO.

\section{Conflict of Interest}

No potential conflict of interest relevant to this article was reported.

\section{References}

1. Habata T, Uematsu K, Hattori K, Kasanami R, Takakura Y, Fujisawa Y. High tibial osteotomy that does not cause recurrence of varus deformity for medial gonarthrosis. Knee Surg Sports Traumatol Arthrosc. 2006;14:962-7.

2. Lobenhoffer P. Importance of osteotomy around to the knee for medial gonarthritis: indications, technique and results. Orthopade. 2014;43:425-31.

3. Akizuki S, Shibakawa A, Takizawa T, Yamazaki I, Horiuchi H. The long-term outcome of high tibial osteotomy: a ten- to 20-year follow-up. J Bone Joint Surg Br. 2008;90:592-6.

4. Bonasia DE, Dettoni F, Sito G, Blonna D, Marmotti A, Bruzzone M, Castoldi F, Rossi R. Medial opening wedge high tibial osteotomy for medial compartment overload/arthritis in the varus knee: prognostic factors. Am J Sports Med. 2014; 42:690-8.

5. DeMeo PJ, Johnson EM, Chiang PP, Flamm AM, Miller MC. Midterm follow-up of opening-wedge high tibial osteotomy. Am J Sports Med. 2010;38:2077-84.

6. Efe T, Ahmed G, Heyse TJ, Boudriot U, Timmesfeld N, Fuchs-Winkelmann S, Ishaque B, Lakemeier S, Schofer MD. Closing-wedge high tibial osteotomy: survival and risk factor analysis at long-term follow up. BMC Musculoskelet Disord. 2011;12:46.

7. Hui C, Salmon LJ, Kok A, Williams HA, Hockers N, van der Tempel WM, Chana R, Pinczewski LA. Long-term survival of high tibial osteotomy for medial compartment osteoar- thritis of the knee. Am J Sports Med. 2011;39:64-70.

8. Koshino T, Yoshida T, Ara Y, Saito I, Saito T. Fifteen to twenty-eight years' follow-up results of high tibial valgus osteotomy for osteoarthritic knee. Knee. 2004;11:439-44.

9. Niinimaki TT, Eskelinen A, Mann BS, Junnila M, Ohtonen P, Leppilahti J. Survivorship of high tibial osteotomy in the treatment of osteoarthritis of the knee: Finnish registrybased study of 3195 knees. J Bone Joint Surg Br. 2012;94: 1517-21.

10. van Raaij T, Reijman M, Brouwer RW, Jakma TS, Verhaar JN. Survival of closing-wedge high tibial osteotomy: good outcome in men with low-grade osteoarthritis after 10-16 years. Acta Orthop. 2008;79:230-4.

11. Saito T, Kumagai K, Akamatsu Y, Kobayashi H, Kusayama Y. Five- to ten-year outcome following medial opening-wedge high tibial osteotomy with rigid plate fixation in combination with an artificial bone substitute. Bone Joint J. 2014;96: 339-44.

12. Bae DK, Song SJ, Yoon KH. Total knee arthroplasty following closed wedge high tibial osteotomy. Int Orthop. 2010;34: 283-7.

13. Sprenger TR, Doerzbacher JF. Tibial osteotomy for the treatment of varus gonarthrosis. Survival and failure analysis to twenty-two years. J Bone Joint Surg Am. 2003;85:469-74.

14. Flecher X, Parratte S, Aubaniac JM, Argenson JN. A 12-28year followup study of closing wedge high tibial osteotomy. Clin Orthop Relat Res. 2006;452:91-6.

15. Gstottner M, Pedross F, Liebensteiner M, Bach C. Long-term outcome after high tibial osteotomy. Arch Orthop Trauma Surg. 2008;128:111-5.

16. Schroter S, Gonser CE, Konstantinidis L, Helwig P, Albrecht D. High complication rate after biplanar open wedge high tibial osteotomy stabilized with a new spacer plate (Position HTO plate) without bone substitute. Arthroscopy. 2011;27: 644-52.

17. Bode G, Schmal H, Pestka JM, Ogon P, Sudkamp NP, Niemeyer P. A non-randomized controlled clinical trial on autologous chondrocyte implantation (ACI) in cartilage defects of the medial femoral condyle with or without high tibial osteotomy in patients with varus deformity of less than 5․ Arch Orthop Trauma Surg. 2013;133:43-9.

18. Staubli AE, De Simoni C, Babst R, Lobenhoffer P. TomoFix: a new LCP-concept for open wedge osteotomy of the medial proximal tibia: early results in 92 cases. Injury. 2003;34 Suppl 2:B55-62.

19. Bae DK, Song SJ, Yoon KH. Closed-wedge high tibial oste- 
otomy using computer-assisted surgery compared to the conventional technique. J Bone Joint Surg Br. 2009;91:1164-71.

20. Gebhard F, Krettek C, Hufner T, Grützner PA, Stockle U, Imhoff AB, Lorenz S, Ljungqvist J, Keppler P; AO CSEG. Reliability of computer-assisted surgery as an intraoperative ruler in navigated high tibial osteotomy. Arch Orthop Trauma Surg. 2011;131:297-302.

21. Lee DC, Byun SJ. High tibial osteotomy. Knee Surg Relat Res. 2012;24:61-9.

22. Cerciello S, Vasso M, Maffulli N, Neyret P, Corona K, Panni AS. Total knee arthroplasty after high tibial osteotomy. Orthopedics. 2014;37:191-8.

23. Noyes FR, Mayfield W, Barber-Westin SD, Albright JC, Heckmann TP. Opening wedge high tibial osteotomy: an operative technique and rehabilitation program to decrease complications and promote early union and function. Am J Sports Med. 2006;34:1262-73.

24. Haslam P, Armstrong M, Geutjens G, Wilton TJ. Total knee arthroplasty after failed high tibial osteotomy long-term follow-up of matched groups. J Arthroplasty. 2007;22:24550.

25. Madan S, Ranjith RK, Fiddian NJ. Total knee replacement following high tibial osteotomy. Bull Hosp Jt Dis. 2002-2003; 61:5-10.

26. Karabatsos B, Mahomed NN, Maistrelli GL. Functional outcome of total knee arthroplasty after high tibial osteotomy. Can J Surg. 2002;45:116-9.

27. Niinimaki T, Eskelinen A, Ohtonen P, Puhto AP, Mann BS, Leppilahti J. Total knee arthroplasty after high tibial osteotomy: a registry-based case-control study of 1,036 knees. Arch Orthop Trauma Surg. 2014;134:73-7.

28. Parvizi J, Hanssen AD, Spangehl MJ. Total knee arthroplasty following proximal tibial osteotomy: risk factors for failure. J Bone Joint Surg Am. 2004;86:474-9.

29. Cameron HU, Park YS. Total knee replacement following high tibial osteotomy and unicompartmental knee. Orthopedics. 1996;19:807-8.

30. Windsor RE, Insall JN, Vince KG. Technical considerations of total knee arthroplasty after proximal tibial osteotomy. J Bone Joint Surg Am. 1988;70:547-55.

31. Noda T, Yasuda S, Nagano K, Takahara Y, Namba Y, Inoue H. Clinico-radiological study of total knee arthroplasty after high tibial osteotomy. J Orthop Sci. 2000;5:25-36.

32. Mont MA, Antonaides S, Krackow KA, Hungerford DS. Total knee arthroplasty after failed high tibial osteotomy. A comparison with a matched group. Clin Orthop Relat Res.
1994;(299):125-30.

33. Meding JB, Wing JT, Ritter MA. Does high tibial osteotomy affect the success or survival of a total knee replacement? Clin Orthop Relat Res. 2011;469:1991-4.

34. Kazakos KJ, Chatzipapas C, Verettas D, Galanis V, Xarchas KC, Psillakis I. Mid-term results of total knee arthroplasty after high tibial osteotomy. Arch Orthop Trauma Surg. 2008; 128:167-73.

35. Haddad FS, Bentley G. Total knee arthroplasty after high tibial osteotomy: a medium-term review. J Arthroplasty. 2000;15:597-603.

36. Bastos Filho R, Magnussen RA, Duthon V, Demey G, Servien E, Granjeiro JM, Neyret P. Total knee arthroplasty after high tibial osteotomy: a comparison of opening and closing wedge osteotomy. Int Orthop. 2013;37:427-31.

37. Toksvig-Larsen S, Magyar G, Onsten I, Ryd L, Lindstrand A. Fixation of the tibial component of total knee arthroplasty after high tibial osteotomy: a matched radiostereometric study. J Bone Joint Surg Br. 1998;80:295-7.

38. Amendola A, Bonasia DE. Results of high tibial osteotomy: review of the literature. Int Orthop. 2010;34:155-60.

39. van Raaij TM, Bakker W, Reijman M, Verhaar JA. The effect of high tibial osteotomy on the results of total knee arthroplasty: a matched case control study. BMC Musculoskelet Disord. 2007;8:74.

40. Efe T, Heyse TJ, Boese C, Timmesfeld N, Fuchs-Winkelmann S, Schmitt J, Theisen C, Schofer MD. TKA following high tibial osteotomy versus primary TKA: a matched pair analysis. BMC Musculoskelet Disord. 2010;11:207.

41. van Raaij TM, Reijman M, Furlan AD, Verhaar JA. Total knee arthroplasty after high tibial osteotomy. A systematic review. BMC Musculoskelet Disord. 2009;10:88.

42. Farfalli LA, Farfalli GL, Aponte-Tinao LA. Complications in total knee arthroplasty after high tibial osteotomy. Orthopedics. 2012;35:e464-8.

43. Meding JB, Keating EM, Ritter MA, Faris PM. Total knee arthroplasty after high tibial osteotomy. A comparison study in patients who had bilateral total knee replacement. J Bone Joint Surg Am. 2000;82:1252-9.

44. Gill T, Schemitsch EH, Brick GW, Thornhill TS. Revision total knee arthroplasty after failed unicompartmental knee arthroplasty or high tibial osteotomy. Clin Orthop Relat Res. 1995;(321):10-8.

45. Young CF, Bourne RB, Rorabeck CH. Tibial tubercle osteotomy in total knee arthroplasty surgery. J Arthroplasty. 2008; 23:371-5. 
46. Whiteside LA. Exposure in difficult total knee arthroplasty using tibial tubercle osteotomy. Clin Orthop Relat Res. 1995; (321):32-5.

47. Zonnenberg CB, Lisowski LA, van den Bekerom MP, Nolte PA. Tuberositas osteotomy for total knee arthroplasty: a review of the literature. J Knee Surg. 2010;23:121-9.

48. Treuter S, Schuh A, Honle W, Ismail MS, Chirag TN, Fujak A. Long-term results of total knee arthroplasty following high tibial osteotomy according to Wagner. Int Orthop. 2012;36: 761-4.

49. Closkey RF, Windsor RE. Alterations in the patella after a high tibial or distal femoral osteotomy. Clin Orthop Relat Res. 2001;(389):51-6.

50. Nagamine R, Inoue S, Miura H, Matsuda S, Iwamoto Y. Femoral shaft bowing influences the correction angle for high tibial osteotomy. J Orthop Sci. 2007;12:214-8.

51. Aglietti P, Buzzi R, Giron F, Zaccherotti G. The Insall-Burst- ein posterior stabilized total knee replacement in the valgus knee. Am J Knee Surg. 1996;9:8-12.

52. Hernigou P, Duffiet P, Julian D, Guissou I, Poignard A, Flouzat-Lachaniette $\mathrm{CH}$. Outcome of total knee arthroplasty after high tibial osteotomy: does malalignment jeopardize the results when using a posterior-stabilized arthroplasty? HSS J. 2013;9:134-7.

53. Akasaki Y, Matsuda S, Miura H, Okazaki K, Moro-oka TA, Mizu-uchi H, Iwamoto Y. Total knee arthroplasty following failed high tibial osteotomy: mid-term comparison of posterior cruciate-retaining versus posterior stabilized prosthesis. Knee Surg Sports Traumatol Arthrosc. 2009;17:795-9.

54. Kleinbart FA, Bryk E, Evangelista J, Scott WN, Vigorita VJ. Histologic comparison of posterior cruciate ligaments from arthritic and age-matched knee specimens. J Arthroplasty. 1996;11:726-31. 